\section{Case Reports in Oncology}

\title{
Subacute Cerebellar Degeneration due to a Paraneoplastic Phenomenon Associated with Metastatic Merkel Cell Carcinoma: A Case Report
}

\author{
Angelos Sharobeam ${ }^{a} \quad J_{a s o n}$ Ray $^{b} \quad$ Juliana Dong ${ }^{a} \quad$ Victor Chong $^{a}$ \\ ${ }^{a}$ Western Health, St Albans, VIC, Australia; ${ }^{b}$ Melbourne Health, Parkville, VIC, Australia
}

\section{Keywords}

Subacute cerebellar degeneration · Merkel cell carcinoma - Metastasis · Paraneoplastic phenomenon

\begin{abstract}
Purpose: The aim of this article is to illustrate the diagnostic challenges and management of paraneoplastic neurological syndromes in Merkel cell carcinoma. Materials and Methods: We describe a previously functionally independent 85-year-old woman who presented with subacute onset of dizziness and gait ataxia in the setting of metastatic Merkel cell carcinoma. Results: Diagnosis was made on biopsy after positron emission tomography imaging revealed increased metabolic activity in 2 left inguinofemoral lymph nodes. Cerebrospinal fluid analysis was positive for anti-Hu on subsequent admission. Her functional status improved with methylprednisolone treatment and radiotherapy. Conclusion: The case highlights the challenge of the evaluation of patients who present with progressive cerebellar signs and the need to consider a paraneoplastic syndrome, especially in the setting of previous malignancy.

(C) 2017 The Author(s)

Published by S. Karger AG, Basel
\end{abstract}


 Oncology}

\section{Introduction}

Paraneoplastic cerebellar degeneration is usually associated with breast, gynaecological and lung cancer, as well as Hodgkin lymphoma [1]. It is characterised by subacute onset of gait instability which may progress to a pan-cerebellar syndrome with vertigo, diplopia, dysarthria, truncal and appendicular ataxia [2] as a consequence of autoimmune mediated destruction of cerebellar Purkinje cells [3].

Several autoantibodies have been associated with paraneoplastic neurological syndromes, with anti-Yo and P/Q type calcium channel antibodies occurring most commonly in cerebellar degeneration. The anti-Hu antibody has also been well characterised in paraneoplastic syndromes, including cerebellar degeneration and encephalomyelitis, amongst others [2]. However, up to $30 \%$ of patients with presumed paraneoplastic syndromes do not have detectable antibodies [4], and a direct pathogenic role of antibodies has only been proven in some paraneoplastic syndromes $[1,2,4]$.

This case highlights the challenges in the evaluation of a patient who presents with a cerebellar syndrome. Paraneoplastic cerebellar degeneration can occur with a wide variety of neoplasms, and there is a wide range of potential causes that need to be considered and excluded.

\section{Case Report}

An 85-year-old woman who lived independently in a retirement village presented in early February 2016 with 10 days of dizziness, nausea and vomiting on a 2-month history of progressively worsening truncal instability and increasing difficulty with fine motor tasks due to incoordination. This resulted in a significant limitation in her personal activities of daily living and mobility.

There were no constitutional symptoms, headache, hearing or visual disturbance. Notably, her past medical history was significant for a previously resected grade 3 Merkel cell carcinoma in January 2015 from her left ankle, for which she declined adjuvant radiotherapy. A sentinel lymph node biopsy was not performed. Other significant history includes excision of a melanoma from her left cheek in 1994 and a left thyroidectomy for a multinodular goitre at age 17 years. There is no known family history of malignancy.

Her initial assessment revealed prominent cerebellar signs, including bilateral upper limb dysmetria and dysdiadokinesis, bilateral lower limb dyssenergia, gait and truncal ataxia. She had normal muscle tone, power, sensation and symmetrical reflexes throughout her upper and lower limbs. Examination of the cranial nerves did not reveal any abnormalities. The remainder of her systems examination demonstrated a left-sided thyroid goitre but was otherwise unremarkable.

Her blood tests, including serum copper levels, electrolytes, autoimmune panel, neural autoantibodies and tumour markers, were normal or negative. Brain and whole-spine magnetic resonance imaging (MRI) scans did not reveal a cause for her symptoms. Her cerebrospinal fluid was negative for autoimmune markers, neural autoantibodies, viral PCR, protein 14-3-3, cytology and electrophoresis.

Thyroid function tests were consistent with a euthyroid state. Anti-thyroid peroxidase (TPO) antibodies were mildly elevated, and thyroglobulin antibody level was within the normal range. 
Computed tomography scan of her chest, abdomen and pelvis revealed an 8.1-mm spiculated lesion in the left lung apex, radiologically felt to be a focus of scarring, and an 11$\mathrm{mm}$ hyperdense left renal upper pole exophytic cortical lesion. She had a multinodular goitre with a dominant nodule measuring $2 \mathrm{~cm}$ in the left lobe of the thyroid. Her positron emission tomography scan revealed 2 metabolically active left inguinofemoral lymph nodes $\left(\mathrm{SUV}_{\max }\right.$ 11.7) consistent with nodal metastases (Fig. 1a, b). There was also an area of moderate to intense uptake in the caecum, suspicious for a tumour. A core biopsy of one of the nodes demonstrated positive synaptophysin staining and weak staining for CD56, consistent with metastatic neuroendocrine carcinoma, likely Merkel cell carcinoma, with a Ki-67 of 80\%.

The leading differential diagnosis for a patient in this age group presenting with a cerebellar ataxia is a cerebrovascular event and as such was our first line of investigation. This was ruled out given the normal brain MRI and the subacute, progressive nature of her symptomatology. Other diagnoses including metabolic, infectious, autoimmune, and metastatic were also considered. The elevated anti-TPO antibody level led to the consideration of Hashimoto encephalopathy as a causative pathology. This was thought unlikely due to her normal conscious state, absence of pyramidal signs [5] and normal cerebrospinal fluid analysis [6].

Infectious aetiologies, such as prion disease, meningoencephalitis and posterior multifocal leukoencephalopathy, were excluded based on normal imaging and cerebrospinal fluid results. A demyelinating disease was thought to be unlikely given the patient's age, progressive nature of her symptoms and normal MRI. Other autoimmune causes, such as systemic lupus erythematosus, Sjögren syndrome and sarcoidosis were excluded based on negative serological markers.

A medication review did not reveal any causative medications to explain her symptoms.Our patient identified returning home and maintaining her independence as the goals of her care.

We commenced a course of $1 \mathrm{~g}$ weekly methylprednisolone for 6 weeks, for the treatment of her likely paraneoplastic syndrome, with improvement in her symptoms. Her treatment was complicated by a single episode of hypomania, which was noted after the second week of methylprednisolone. Other potential therapies, including intravenous immunoglobulin, were considered but not administered, given her positive response to methylprednisolone.

The patient and her family decided that she would not undergo systemic therapy. She subsequently received a total of $45 \mathrm{~Gy}$ of radiotherapy to the left external iliac nodes and small bowel for locoregional and symptom control.

She improved from a baseline of requiring the assistance of 2 people for mobility and transfers to mobilising short distances with mobility aids under supervision. Her care was transferred to a subacute transitional care program, and she was able to return home at her baseline level of function.

She was admitted 5 weeks later with a several-day history of upper and lower limb ataxia and difficulty mobilising. Repeat anti-neuronal antibody testing was positive for anti-Hu antibody. Another 1-g dose of methylprednisolone was given with subsequent improvement in her symptoms, and she continues on monthly 1-g doses. 


\section{Discussion}

Merkel cell carcinoma is a rare, aggressive cutaneous neuroendocrine carcinoma of older patients with a propensity for local and regional recurrence [7]. Metastases to other sites, including lung, bowel, bone and the central nervous system, have also been reported [8, 9].

Paraneoplastic phenomena associated with Merkel cell carcinoma are uncommon [10, 11], and we are aware of only 2 previously published cases of paraneoplastic cerebellar syndromes associated with Merkel cell carcinoma [10,11].

Zhang et al.'s [10] patient developed cerebellar syndrome with P/Q type voltage-gated calcium channel (VGCC) antibodies in the cerebrospinal fluid. The patient improved significantly after tumour excision and aggressive immunotherapy.

Iyer et al. [11] reviewed 663 cases of Merkel cell carcinoma over a 24-year period, one of whom had an acute cerebellar syndrome [12]. They describe a patient with acute onset of gait instability, incoordination and an enlarged iliofemoral node, histologically consistent with Merkel cell carcinoma. There were no detectable anti-neuronal antibodies. The patient had resolution of their symptoms after tumour excision and adjuvant radiotherapy, extending to a follow-up period of 7 years.

Merkel cell carcinoma has also been associated with Lambert-Eaton myasthenic syndrome with anti-VGCC $[11,13]$ and anti-Hu positivity [14]. VGCC autoantibody positivity has been associated with Lambert-Eaton myasthenic syndrome from small cell lung cancer [15, 16], which is the most common form of neuroendocrine tumour associated with a paraneoplastic neurological process [17]. Anti-Hu antibodies have been associated with cerebellar degeneration in other forms of malignancy [18], but not Merkel cell carcinoma, prior to this case.

Paraneoplastic cerebellar degeneration may occur with rare neuroendocrine tumours, such as Merkel cell carcinoma, and clinicians should investigate for malignancy in patients presenting with a subacute cerebellar syndrome. The low sensitivity and specificity of identified neuronal autoantibodies means that clinicians must retain a high index of suspicion even with an initially negative result, and a highly suggestive clinical presentation, exclusion of alternate pathology and response to immunotherapy should support a presumptive diagnosis. Furthermore, this case highlights the importance of interpreting test results in the clinical context; our patient had positive anti-TPO antibodies, but no other features of Hashimoto thyroiditis, to support an alternate diagnosis.

\section{Statement of Ethics}

The authors have no ethical conflicts to declare.

\section{Disclosure Statement}

The authors have no conflicts of interest to disclose.

\section{Funding Sources}

No external or internal funding or support was required for this case report. 


\section{Case Reports in Oncology}

\begin{tabular}{l|l}
\hline Case Rep Oncol 2017;10:764-768 \\
\hline DOI: 10.1159/000479731 & $\begin{array}{l}\text { (c) 2017 The Author(s). Published by S. Karger AG, Basel } \\
\text { www.karger.com/cro }\end{array}$ \\
\hline
\end{tabular}

Sharobeam et al: Subacute Cerebellar Degeneration due to a Paraneoplastic Phenomenon Associated with Metastatic Merkel Cell Carcinoma: A Case Report

\section{References}

1 Rees JH: Paraneoplastic syndromes: when to suspect, how to confirm, and how to manage. J Neurol Neurosurg Psychiatry 2004;75:ii43-ii50.

2 Rosenfeld MR, Dalmau J: Update in paraneoplastic neurologic disorders. Oncologist 2010;15:603-617.

-3 Mitoma H, Adhikari K, Aeschlimann D, et al: Consensus paper: neuroimmune mechanisms of cerebellar ataxias. Cerebellum 2016;15:213-232.

- 4 Honnorat J, Antoine JC: Paraneoplastic neurological syndromes. Orphanet J Rare Dis 2007;2:22.

$\checkmark 5$ Zhou JY, Xu B, Lopes J, et al: Hashimoto encephalopathy: literature review. Acta Neurol Scand 2017;135:285-290.

-6 Kothbauer-Margreiter I, Sturzenegger M, Komor J, et al: Encephalopathy associated with Hashimoto thyroiditis: diagnosis and treatment. J Neurol 1996;243:585-593.

-7 Tothill R, Estall V, Rischin D: Merkel cell carcinoma: emerging biology, current approaches, and future directions. Am Soc Clin Oncol Educ Book 2015;35:e519-e526.

8 Matkowskyj KA, Hosseini A, Linn JG, et al: Merkel cell carcinoma metastatic to the small bowel mesentery. Rare Tumors 2011;3:e2.

-9 Zhan FQ, Packianathan VS, Zeitouni NC: Merkel cell carcinoma: a review of current advances. J Natl Compr Canc Netw 2009;7:333-339.

10 Zhang C, Emery L, Lancaster E: Paraneoplastic cerebellar degeneration associated with noncutaneous Merkel cell carcinoma. Neurol Neuroimmunol Neuroinflamm 2014;1:e17.

11 Iyer JG, Parvathaneni K, Bhatia S, et al: Paraneoplastic syndromes (PNS) associated with Merkel cell carcinoma (MCC): a case series of 8 patients highlighting different clinical manifestations. J Am Acad Dermatol 2016;75:541-547.

12 Balegno S, Ceroni M, Corato M, et al: Antibodies to cerebellar nerve fibres in two patients with paraneoplastic cerebellar ataxia. Anticancer Res 2005;25:3211-3214.

-13 Eggers SD, Salomao DR, Dinapoli RP, et al: Paraneoplastic and metastatic neurologic complications of Merkel cell carcinoma. Mayo Clin Proc 2001;76:327-330.

$\checkmark 14$ Lopez MC, Pericay C, Agusti M, et al: Merkel cell carcinoma associated with a paraneoplastic neurological syndrome. Histopathology 2004;44:623-630.

-15 Bekircan-Kurt CE, Ciftci ED, Kurne AT, et al: Voltage gated calcium channel antibody related neurological diseases. World J Clin Cases 2015;3:293-300.

-16 Titulaer MJ, Lang B, Verchuuren JJ: Lambert-Eaton myasthenic syndrome: from clinical characteristics to therapeutic strategies. Lancet Neurol 2011;10:1098-1107.

17 Kaltsas G, Androulakis II, Herder WW, et al: Paraneoplastic syndromes secondary to neuroendocrine tumours. Endocr Relat Cancer 2010;17:173-193.

18 Graus F, Delattre JY, Antoine JC, et al: Recommended diagnostic criteria for paraneoplastic neurological syndromes. J Neurol Neurosurg Psychiatry 2004;75:1135-1140.
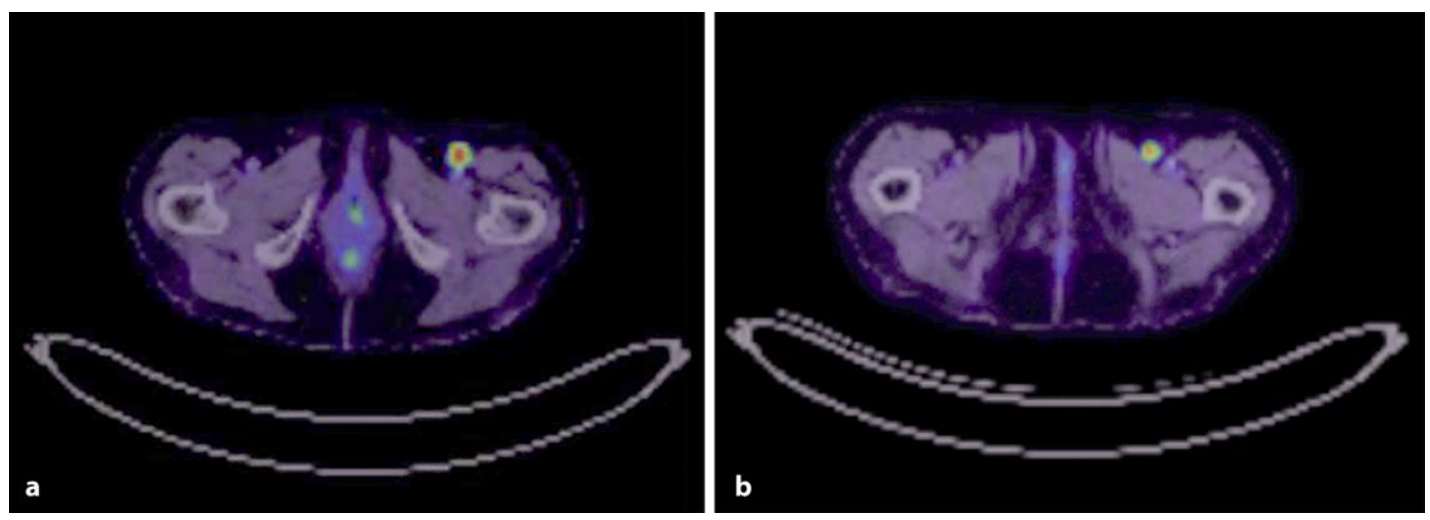

Fig. 1. a, b Left inguinofemoral node metastasis. 